\title{
The psi operon of Rhizobium leguminosarum biovar phaseoli: identification of two genes whose products are located at the bacterial cell surface
}

\author{
M. L. Mimmack, ${ }^{1}$ D. Borthakur, ${ }^{2}+$ M. A. Jones, ${ }^{1}$ J. A. Downie ${ }^{2}$ \\ and A. W. B. Johnston'
}

Author for correspondence: A. W. B. Johnston. Tel: +44603 592264. Fax: +44603592250.

1 School of Biological

Sciences, University of East Anglia, Norwich NR4 7TJ,

UK

2 John Innes Institute, Colney Lane, Norwich NR4 7UH, UK

\begin{abstract}
We have delineated three short open reading frames, psiA, ORF-P and psiB within the psi operon of Rhizobium leguminosarum biovar phaseoli. psiA, in a multi-copy plasmid, causes inhibition of exopolysaccharide synthesis in $R$. leguminosarum. In addition, the suppression of exopolysaccharide synthesis due to the multi-copy psiA caused $R$. leguminosarum strains to stain with the dye calcofluor, a response that does not occur with wild-type strains of this species. Insertions of a defective phoA gene (lacking its promoter, ribosomal binding site and leader sequence) into psiA and psiB were isolated and the precise locations of the insertions were established. PsiA-PhoA and PsiB-PhoA protein fusions were found to express alkaline phosphatase activity indicating that PsiA and PsiB span the inner membrane or are translocated across it.
\end{abstract}

Keywords: Calcofluor, exopolysaccharide, pho A-fusions, Rbizobium

\section{INTRODUCTION}

Strains of the bacterial genera Rbizobium, Bradyrhizobium and Azorbizobium induce nitrogen-fixing nodules on legumes. The particular species of legume that is nodulated by a given species or strain of Rbizobium is determined by a suite of nodulation (nod) genes which has, in several cases, been located on large 'symbiotic' plasmids, close to other genes required for symbiotic nitrogen fixation (Downie \& Johnston, 1988).

Many of these nod genes are involved in the synthesis of signal lipooligosaccharides that induce early steps in nodulation (see Fisher \& Long, 1992). In addition to these nod genes, other Rbizobium genes are necessary for successful nodulation. Notably, it has been shown that the ability to produce the high molecular mass acidic exopolysaccharide (EPS) is required for the induction of nitrogen-fixing nodules. For example, R. meliloti mutants

† Present address: Biotechnology Program, University of Hawaii, 3050 Maile Way, Honolulu, Hawaii 96822, USA.

Abbreviations: AP, alkaline phosphatase; EPS, exopolysaccharide; NTG, nitrosoguanidine; X-P, 4-chloro-5-bromo-3-indolyl phosphate.

The GenBank accession number for the sequence reported in this paper is L20651. which fail to make the succinoglycan EPS (exo) induce nodules on alfalfa, but the nodules are devoid of bacteria and consequently fail to fix nitrogen (Leigh $\mathrm{et}$ al., 1985). In $R$. leguminosarum bv. viciae, EPS deficient mutants fail to induce nodules on peas (Borthakur et al., 1986), but such mutants can induce nitrogen-fixing nodules on determinate nodulating plants such as Phaseolus beans (Borthakur et al., 1986; Hotter \& Scott, 1991).

In $\mathrm{R}$. meliloti, where the most detailed genetic analyses have been conducted (see Leigh \& Coplin, 1992; Gray \& Rolfe, 1990), most of the exo genes are located in a cluster on a large indigenous plasmid, different from the ' $\mathrm{pSym}$ ' that contains the nod and nif genes (Long et al., 1988; Finan et al., 1986; Hynes et al., 1986). However, a gene was found located close to several nod and nif genes which could have marked effects on EPS synthesis in a strain of R. leguminosarum bv. phaseoli, (Borthakur et al., 1985; Lamb et al., 1985; Davis \& Johnston, 1990a,b; Hawkins $\&$ Johnston, 1988). This gene was termed psiA (polysaccharide inhibition) and was detected initially because Rbizobium strains containing the cloned psi gene were non-mucoid. These EPS ${ }^{-}$strains were defective in nodulation of peas and Phaseolus beans by strains of $R$. leguminosarum biovars viciae and phaseoli, respectively (Borthakur et al., 1985), and a strain carrying a psi $A:: \operatorname{Tn} 5$ mutation induced on Phaseolus beans non-fixing nodules 
which contained few, if any, bacteria (Borthakur et al., 1985; Latchford et al., 1991).

psi $A$ is predicted to encode a small $\left(M_{\mathrm{r}} 10000\right)$ protein with a strongly hydrophobic $\mathrm{NH}_{2}$-half and a hydrophilic carboxy-domain, suggesting that it may be anchored in the cytoplasmic membrane of the bacteria (Borthakur \&: Johnston, 1987). Further, some translational fusions between $p s i A$ and a defective $p h o A$ gene that does not encode a signal peptide sequence were found to express alkaline phosphatase (AP) activity, providing evidence that $p$ si $A$ encodes a protein which transverses the bacterial inner membrane (Latchford et al., 1991).

Bacteroids make little or no EPS (Tulley \& Terry, 1985) and it was proposed (Borthakur $e t$ al., 1985) that a normal function of psiA was to shut down EPS synthesis by bacteroids; indeed, $p s i A$ is expressed at high levels in bacteroids but, normally, only weakly in free-living $R$. leguminosarum bv. phaseoli (Latchford et al., 1991). It was suggested that increased levels of Psi protein resulting from the cloned psi $A$ could inhibit EPS synthesis in freeliving Rbizobium.

Genetic evidence indicated that psi $A$ was part of a longer transcriptional unit. Recombinant plasmids containing psi $A$, plus as much as $1 \mathrm{~kb}$ upstream of the gene, only conferred a $\mathrm{Psi}^{+}$phenotype if the cloned DNA was transcribed from a constitutive promoter in the cloning vector. However, if the cloned DNA extended to $1.3 \mathrm{~kb}$ upstream of $p s i A$, the inhibitory effects of cloning this region were independent of the orientation. There is good evidence that this $1 \mathrm{~kb}$ region contains at least one other gene since Latchford et al. (1991) isolated translationally active $p h o A$ insertions within it.

Transcription of the $p s i$ region is repressed by the gene, psr $A$, which is about $13 \mathrm{~kb}$ from psi $A$ on the symbiotic plasmid pRP2JI (Borthakur \& Johnston, 1987). The predicted sequence of PsrA revealed an $\alpha$ helix-turn- $\alpha$ helix motif, which has striking similarity to the DNAbinding domains of several other prokaryotic transcriptional regulators (Mimmack et al., 1994). Introducing a plasmid carrying $\operatorname{psr} A$ restored EPS production to a strain containing multi-copy psi $A$ (Bortkahur \& Johnston, 1987).

By a very different mechanism, the cloning of another gene, termed $p s s$, also suppressed the EPS ${ }^{-}$phenotype conferred by multi-copy psi $A$ (Borthakur et al., 1988). pss $A$ is required for EPS synthesis (Borthakur $e$ t al., 1986) and, like $p s i A$, specifies a membrane-associated protein. $p s i A$ does not affect the transcription of $p s s A$ and $p s s A$ does not affect the transcription of psi $A$ (Borthakur $e t$ al., 1988). It was suggested that there is an association between the PsiA and PssA proteins in the membrane and an excess of PsiA relative to PssA causes the failure to make EPS; but, when pss $A$ itself is also cloned, the correct 'balance' between PsiA and PssA is restored, allowing EPS synthesis (Borthakur et al., 1986).

A gene (termed exoX) whose behaviour is similar to that of psi $A$ was later identified in R. meliloti and Rhizobium sp. NGR234 (Reed et al., 1991; Gray et al., 1990). As with psi $A$, cloning of exoX causes a dominant $\mathrm{EPS}^{-}$phenotype in rhizobia. There are structural similarities in the ExoX and PsiA proteins; they have very similar sizes and almost superimposable hydropathy profiles, but amino acid sequence identity between Exo X and PsiA is restricted to a short (14 amino acid) tract close to the junction of the hydrophobic and hydrophilic portions of the proteins (see Gray et al., 1990). The ability of exoX to inhibit EPS synthesis can be overcome by the presence of multiple copies of another gene, exoY (Reed et al., 1991; Gray et al., $1990)$ which has a significant sequence identity to $p s s A$ (see above). The model put forward (Gray \& Rolfe, 1990) for the way in which ExoY overcomes multi-copy exoX was very similar to that suggested by Borthakur et al. (1986) for the interaction between PsiA and PssA.

In this paper we present the sequence of the psi region, describe the structure of $p s i B$, and identify the precise locations of the 'active' $p s i-p h o A$ fusions. Further, we describe a surprising phenomenon which relates the presence of psiA to the ability of Rbizobium colonies to stain with the optical brightener calcofluor.

Table 1. Bacterial strains and plasmids

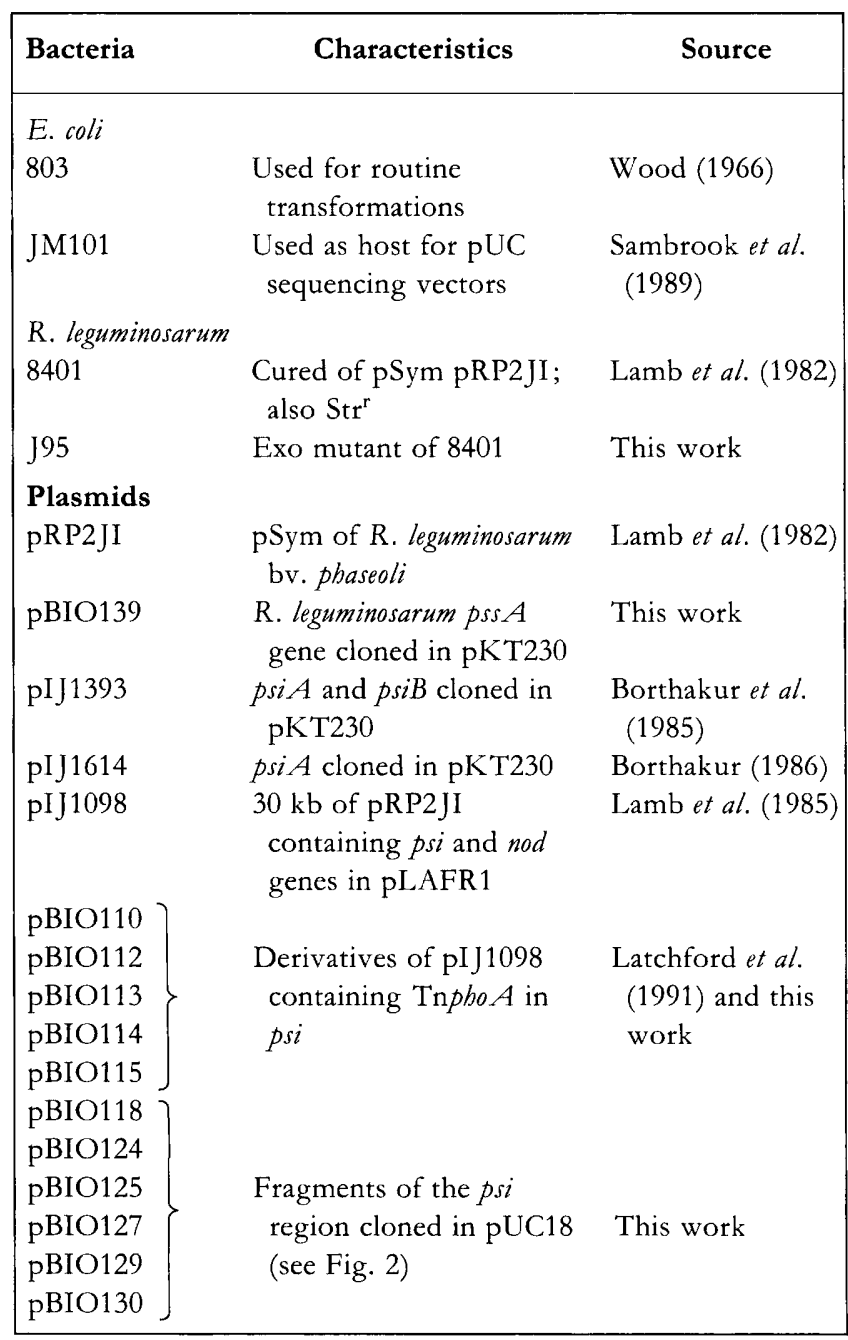




\section{METHODS}

Bacterial strains and plasmids. These are shown in Table 1.

Media and general growth conditions. These were as described by Beringer (1974). 4-Chloro-5-bromo-3-indolyl phosphate sodium salt (X-P) was incorporated into solid media at $40 \mu \mathrm{g} \mathrm{ml}^{-1}$ and, where appropriate, $0.02 \%$ (w/v) calcofluor (Sigma). Triparental 'patch cross' matings were done as described by Buchanan-Wollaston et al. (1980) using pRK2013 as the 'helper' plasmid.

Isolation of EPS mutants of $\boldsymbol{R}$. leguminosarum. Approximately $10^{8}$ cells of $R$. leguminosarum were spread on a plate containing TY medium. After $4 \mathrm{~h}$ at $28{ }^{\circ} \mathrm{C}$, a few crystals of nitrosoguanidine (NTG) were placed in the centre of the plate. After $3 \mathrm{~d}$ at $28^{\circ} \mathrm{C}$ there was a zone of clearing around the NTG, from the edge of which cells were scraped off with a sterile loop, resuspended in $20 \%$ glycerol and plated out at appropriate dilutions on TY medium. Non-mucoid colonies were identified after $3 \mathrm{~d}$ of growth at $28^{\circ} \mathrm{C}$.

DNA manipulations and sequencing. Routine DNA manipulations (restriction digestions, ligation transformation, etc.) were done essentially according to Sambrook et al. (1989). DNA sequencing of the $1700 \mathrm{bp}$ psi region was done by compiling a restriction map of this fragment, aided, in part, by the knowledge of some sequences upstream of psi $A$ (Borthakur, 1986). Appropriate fragments were cloned into pUC18 vectors and double-stranded DNA sequencing was done using the Sanger dideoxy method. Sequence data were obtained and compiled using an automated ALF (Pharmacia) sequencer according to the manufacturer's instructions. To sequence the junctions between $p h o A$ and the $p s i$ genes, an oligonucleotide, KH107, corresponding to the $5^{\prime}$ end of the $p b o A$ gene was synthesized, purified by standard methods and used as a primer in sequencing reactions.

\section{RESULTS}

\section{Multicopy psi causes $R$. leguminosarum to stain with the optical brightener calcofluor}

Much of the work on exo genetics in R. meliloti has hinged upon the observation that the EPS of this species stains with the optical brightener calcofluor when the colonies are viewed under UV light; mutations in different exo loci variously abolish or reduce this staining (see Leigh \& Coplin, 1992). In fact, colonies of several strains of $R$. meliloti do not have a mucoid appearance, and exo mutants were isolated by their failure to fluoresce. For reasons which are not clear, colonies of $R$. leguminosarum do not fluoresce with calcofluor under UV, despite their pronounced mucoid appearance and we have failed to observe staining with the dye with several wild-type strains and biovars of this species both on complete (TY) and minimal (Y) media (unpublished observations).

During the course of this survey, we examined the calcofluor staining of $R$. leguminosarum strains and mutants and came across what appeared to be a paradoxical result. A derivative of $R$. leguminosarum harbouring the plasmid pIJ1393 (which contains $p$ si $A$ plus upstream sequences) fluoresced when examined under UV light (Fig. 1) after growth on TY medium supplemented with calcofluor. This was due to the $p$ siA gene since $R$. leguminosarum strain 8401 carrying another plasmid, pIJ1614, containing only psi $A$ cloned in pKT230 also fluoresced under LV light after growth with calcofluor.

Thus, the presence of psiA conferred on R. leguminosarum the ability to make (or to expose) calcofluor-staining material. To test if this required the formation of the native EPS, a wild-type strain of $R$. leguminosarum bv. phaseoli was mutagenized with NTG (see Methods).
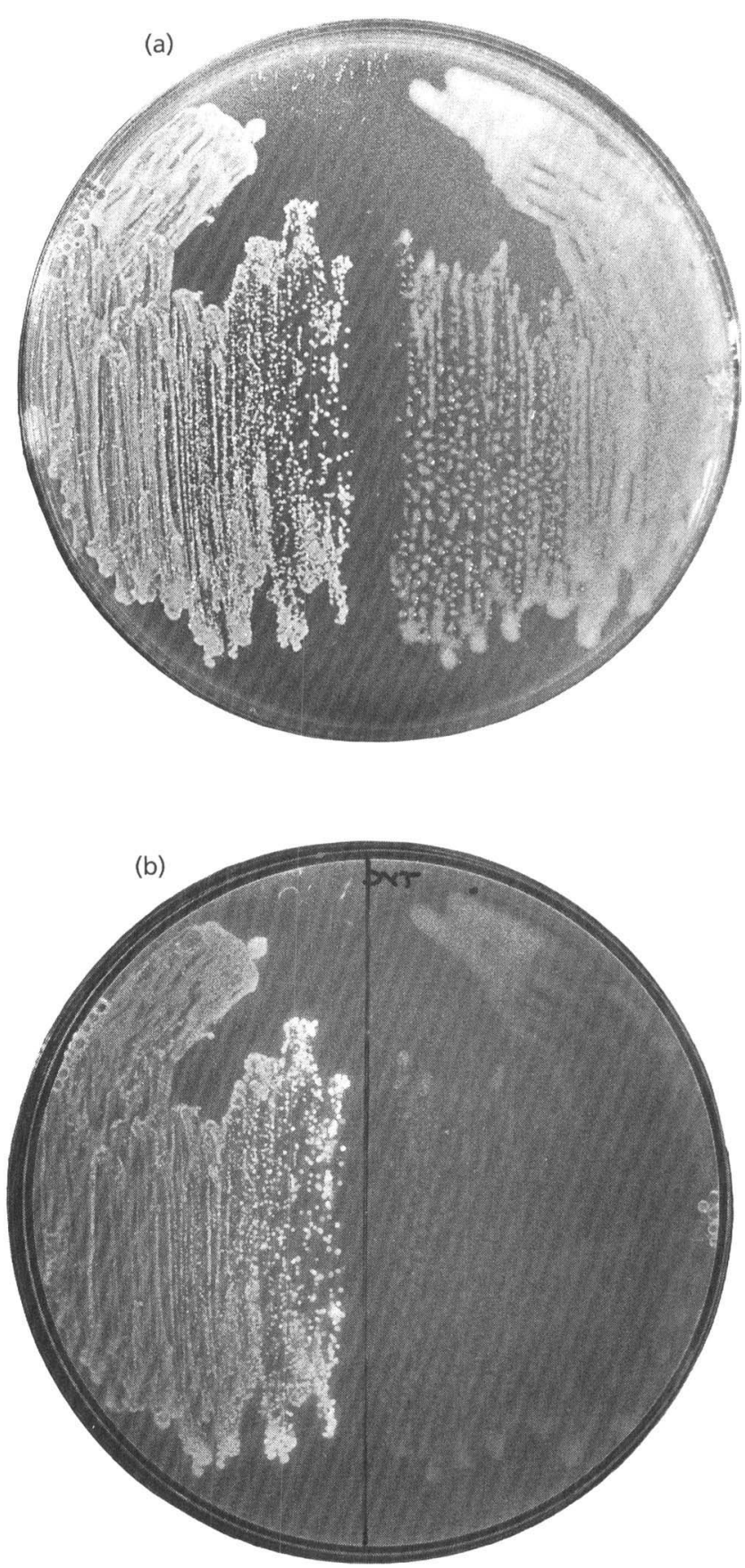

Fig. 1. Fluorescing colonies of psi-containing strains. Strains of 8401 (to the right in each plate) and 8401(pIJ1393) (to the left) were streaked on TY medium and were photographed under visible (a) and UV (b) light. 

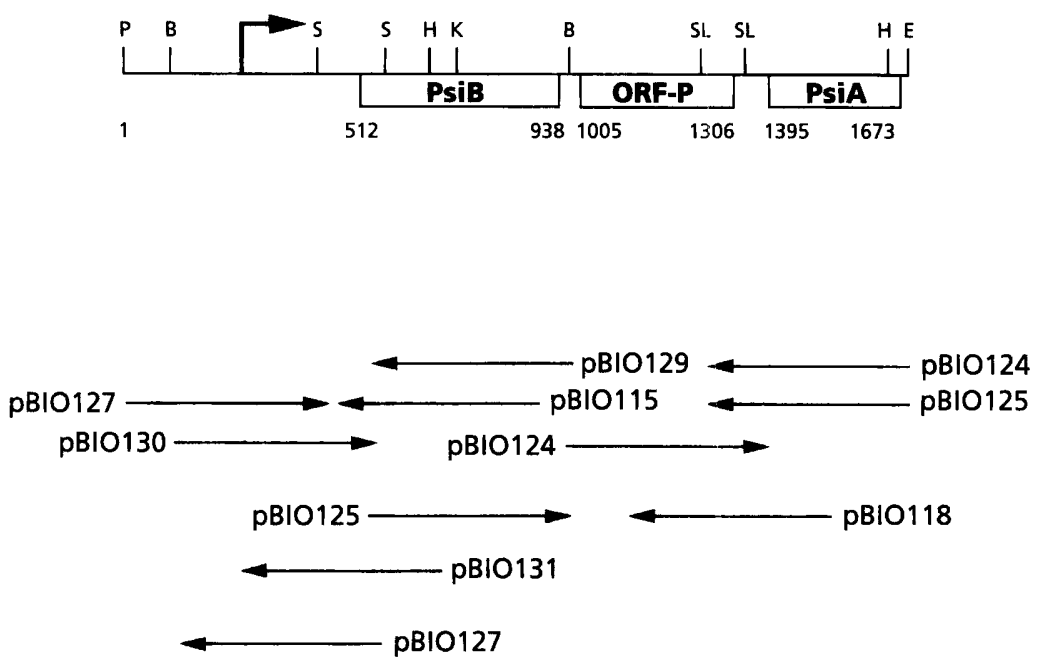

Fig. 2. Representation of the psi region. Dimensions of the psiA and psiB genes and location of ORF-P. Restriction sites are for Pstl (P) BamHI (B), SaCl (S), HindIII (H), KpnI $(\mathrm{K})$ and Sall (S). The boxed area above the map represents the previously sequenced psiA gene (Borthakur \& Johnston, 1987). Various fragments were cloned into Bluescript to form the ' $\mathrm{pBIO}$ ' plasmids which were sequenced in the orientation indicated. The thick arrow indicates the direction of transcription of the psi genes.

Out of several thousand surviving colonies, four were identified which were non-mucoid on TY medium. Each of these was shown to be complemented to $\mathrm{EPS}^{+}$ by $\mathrm{pBIO} 139$ which carries the pss $A$ gene cloned in pKT230 (pBIO139 was made by first cloning a $1076 \mathrm{bp}$ Eco RV-HindIII fragment containing pss $A$ into pUC18 and then releasing pss $A$ as an EcoRI-SacI fragment before ligating to $\mathrm{pKT} 230$ ). Therefore, these four mutants appear to be affected in the pss $A$ gene. One of these mutants (J95) was then used as a recipient for the introduction of pIJ1393 ( $p s i$ ). Not surprisingly, the transconjugants were non-mucoid. When plated on T'Y medium supplemented with calcofluor and examined under UV light, the colonies failed to fluoresce indicating that the production of the calcofluor-staining material in strains containing multi-copy psi requires the function of at least one pss (exo) gene.

\section{Sequencing the psi transcriptional unit}

Previously (Borthakur \& Johnston, 1987), it had been shown that a cloned $1.7 \mathrm{~kb}$ Pst I-EcoRI fragment inhibited EPS production irrespective of its orientation relative to the promoter in the vector and, therefore, this fragment appeared to contain both the $p s i A$ gene and its promoter. In addition, it contained the gene in which translationally active $p h o A$ fusions had been identified (Latchford $e t$ al., 1991). The DNA sequence of the psi $A$ gene had been determined (Borthakur \& Johnston, 1987). To complete the sequencing of the region $5^{\prime}$ of psi $A$ a detailed restriction map was compiled (aided in part by access to sequence information obtained by Borthakur, 1986) and various fragments were cloned into pUC18 prior to sequencing (Fig. 2). Analysis of the region $5^{\prime}$ of psiA revealed two significant ORFs in the same orientation as psi $A$, both with a high probability of encoding proteins (Fig. 3). The upstream ORF was confirmed to be a gene on the basis of protein fusions (see below) and was termed $p s i B$. The intervening ORF was termed ORF-P.
The proposed translational starts of $p s i B$ and ORF-P were chosen on the basis of their being preceded by satisfactory ribosomal binding site sequences. Analysis of $p s i B-p h o A$ fusions shows that the translational start of $p s i B$ must lie upstream of position 588 (see below). Therefore, $p s i B$ is likely to start at the GTG(V) at 537 or the TTG(L) at 527 . The deduced amino acid sequences of $p s i B$ and ORF-P were compared with those in databases; no significant similarities to any known sequence were found.

Previously, genetic evidence had shown that the psi promoter lies between the Bam HI site at position 109 and the SacI site at position 437 (Borthakur \& Johnston, 1987). This region was examined for any consensus promoter sequences, regions of dyad symmetry or repeats, but none was found.

\section{Sequencing the psi-phoA junctions}

The recombinant plasmid pI 1098 contains $30 \mathrm{~kb}$ of pRP2JI, including $p s i A$ and $B$. Derivatives of pIJ1098, containing $\operatorname{Tn} 5-p h o A$ insertions which abolished $p s i$ activity, while expressing AP had been isolated (Latchford et al., 1991). It was noted that on X-P supplemented media, colonies containing the (probably clonal) in sertions in pBIO110 and $\mathrm{pBIO} 114$ were brighter blue than those in the other three fusion plasmids pBIO113, pBIO115 and pBIO112. To sequence the junctions, the Tn 5 - pho $A$ fusion sites from these five plasmids were subcloned by digesting the plasmids with BamHI (which cuts within Tnpbo A), ligating the fragments with pUC18 and selecting $\operatorname{Kan}^{\mathrm{r}}$ transformants. Such fragments contained the aph gene (kan), pho $A$ plus the part of the $p s i$ region extending to the next $5^{\prime} \mathrm{BamHI}$ site.

Using a pho $A$-specific oligonucleotide as a sequencing primer (see Methods) the locations of the pho As were established (Fig. 3). In four cases (two of whichpBIO110 and pBIO114 - are likely to be clonal) pho $A$ was 
at I

C'TGCAg CATGgGAGATGAT'TCCAATTCTGCCGgGOTATTCGTACAGCGTACAGCTCTCGTCAAACTTGGAACACGCACCCGTTTGCATAGCCTGCCCAA IOO

Bamin I

101 GGTCGGGATCCCGTTCCGCAAATTTGGGACAAATGTCGAGCCATACGAGCGGCGACGCGATTGGGCCGCCACGTTCGATTGCTGAACTCGCCTTAGTCAC 200

201 TGCGCGGCTGCTGGTATGGTCAACATCGTCGCGTACATGTTIGGCGCGATTGGTATGGGGGCGCGCGGATAAACCACGGTATCAATATCTAATAAAGTCA 300

301 ACGATATGGCGACATTGAGCGACACGTCCGGAGCGTGACTTGAATTGAACCGAACCGAATTGGCGCAGGACCATTTACACAGCACGCTAAATCTGCATAT 400

Bao I

401 TGCCGCGCGTTGTCGCTATCCCGAGTTGATGCACGAGCTCTT"TCGCTCCCGTCAAAAGCAGCTGGGCACGGGCTCTMTTCAAGAATTTAGAAGGCAAGGA 500

sao I pBIOL10,pBIOL14>

501 TATGGAAAATTTGTGTATCTGTTGGAGCCGGAAAGTGCGATCCTTTCGCGCCGCTGAGCTCCCTGATCGCAACAGCATTGCATCGATCTCCGGTTTAAT 600

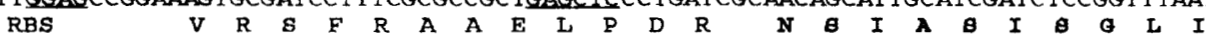

Hind II I

601 TGGCAGTGATCTTATTCAGATGATCCGCTTCGACGACATGCACTCGCTATTTGTTGGGGAAGAAGCTTTGCGAGTTGGGTTAACCGCCTTCACGATCTTT 700

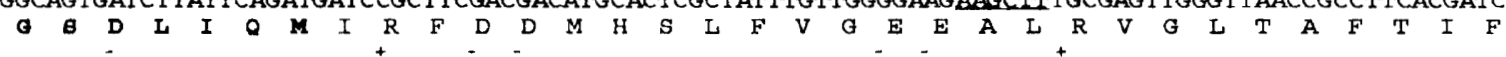

xpon I

PBI0113>

701 GACGGGTACCCGATCCCTCTTGCCGGGCAGATAGCGCTTTTGGGAGGCGACGGTAGTAAACCTTATCGTTCACCGTCAATAACGATGACGGAAGCCGCGC 800

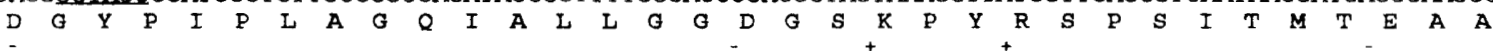

801 GACGTTTTGAATGTTGCCGGCCGGTGCTTGATCCTGTGTTTGCTCCGATGGACCGAGTGGCGAACAAGGGCCTCATCGTTGCGGGCGCACTGGAAAGCCT 900

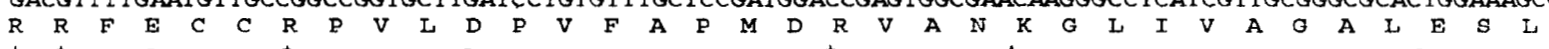

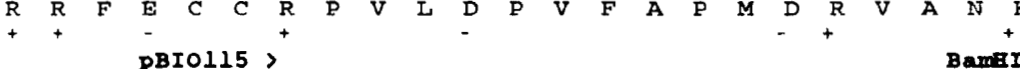

901 GCAAGTGCGTATTGATCGCCGCTCCCCGGTGCTCCTATGAGCGCAATGACGAATGATAGGATCCAGGTCGCGCCGATTCGCTCAGAGTCCGCGAGTGTGC 1000

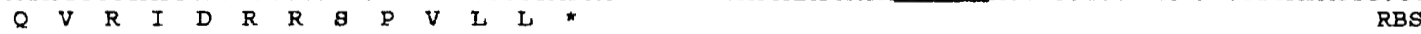

1001 CGACATGGGCTGGAGAAACGGAAAGCTGACGCCTCAAAGCGGCGTTCACTACGTTGCCGAAGGCCTCCCGTTCAACTGGACAAAGGTCAACACTGAAGGG 1100

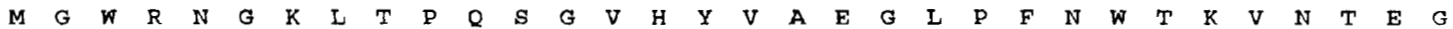

1101 CGGAAGACGTTCAGTGCATTTGCCGAGTTGGAAGCCGCGAAGGAAAAAGTCGCCATTTTGGCTGTTCGCGCTGACCCGGCT"TTGGCAACCTATGGCACC 1200

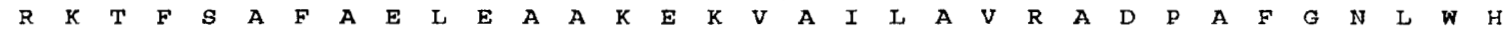

\section{Dal I}

1201 AGCTGAATOCCGAAGTAGTTGATCGCTGTCGACGGCGCAACCCGCTWTGCATGCAGACGCTGTTTCAACGCCTGCTCTCGGCGAAGTCATGCACGTTCAT 1300

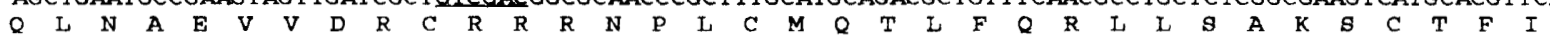

Bal I

1301 AGCGTAGACGAACCGGGCGAAATCATCGGCGTCGACGCAGTTGAAACATTGGACGAACGAACGGGAGCAAGACCTTGCCCTTTAGAACCTTTTTTTGCAT 1400 $A \stackrel{0}{*}$

1401 CCTGCTGCTGGTCACGTGCACCAGCGCTGTTTCGGCCTACGCGCCTCGCTTTCCATCITCAAAGCGTTCGCCGTAACCTTAGCGGCTTCGGTTTTCCTTC 1500

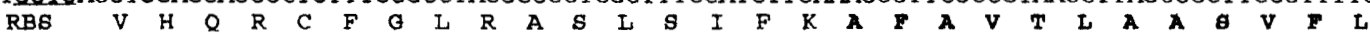

1501 AGGTCGTTTATTTCTTGAGCCTGCTATTTATGTCCTTCCGGCCCACGCGCGAGAGCGACAGATCGATACATTCAGGAACTCGGCAGGCAGACCAGCCACA 1600

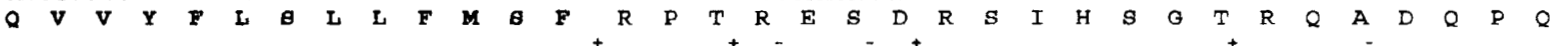

Hina III BOORI

1601 AAAACGTGATCGCGATAAAACAGAGCAGTCCAACGTCCCCAAGCTTGACCCAAGGCGTAAACGGCGCACCCCTTAGCGGTGTAGGTATTGACCGAATTC 1699 $\begin{array}{lllllllllllllllllllllllllll}K & R & D & R & D & K & T & E & Q & B & N & V & P & K & L & D & P & R & R & K & R & R & T & P & *\end{array}$

Fig. 3. DNA sequence of the psi region. psiB extends from 537-936, ORF-P from 1006-1305 and psiA from $1420-1670$. Relevant restriction sites are shown as are potential ribosomal binding sites (RBS). The locations and corresponding plasmids of the fusions which expressed AP are each marked by $a^{\prime}$ ' $>$ '. In all cases, transcription of the phoA was from left to right. Potential membrane-spanning regions are shown in bold and the positions of charged amino acids are indicated.

fused in frame with $p$ siB and one was in $p s i A$. In all cases, the insertions were in the expected orientation.

\section{DISCUSSION}

In the work presented here, we have identified and sequenced a gene, $p s i B$, upstream of the previously studied psi. $A$ gene of $\mathrm{R}$. leguminosarum bv. phaseoli. Between psi $A$ and $p s i B$ is another ORF in the same orientation, but it is yet to be shown formally that ORF-P does represent a gene. The deduced polypeptide products of ORF-P and $p s i B$ showed no similarity to any sequences in databases that were interrogated, and there is, at present, no understanding of their biochemical functions.

Insertion mutations into $p s i B$ and ORF-B have been obtained (Latchford et al., 1991; Borthakur, 1986). Since these insertions would be polar on $p s i A$, it is not surprising that their phenotypes mimic those of mutations into psiA itself, i.e. loss of the ability to inhibit EPS 
synthesis when present in multi-copy plasmids and $\mathrm{Fix}^{-}$ on beans when introduced by marker exchange into pRP2JI (Latchford et al., 1991). Thus, the specific effects of non-polar mutations into psiB or into ORF-P remain to be established.

Based on the hydrophobic nature of the PsiA protein it had been suggested that it was located in the cytoplasmic membrane of Rbizobium (Borthakur \& Johnston, 1987). The single 'active' PsiA-AP fusion was at the $\mathrm{NH}_{2}$-end of PsiA (see Fig. 3) only six amino acids downstream of the Val that was originally suggested by Borthakur \& Johnston (1987) to be the $\mathrm{NH}_{2}$-terminus. In psi $A$ there is another potential start codon (TTG) $21 \mathrm{bp}$ upstream of the $\mathrm{Val}(\mathrm{GTG})$. In either event this extreme $\mathrm{NH}_{2}$-region is not itself hydrophobic nor were there any similarities to consensus leader sequences that would translocate the protein into the periplasmic space.

The four $p s i B-p h o A$ fusions (two of which are likely to be clonal) were scattered throughout the gene. Analysis of the deduced product of $p s i B$ revealed the presence of one potential membrane-spanning region near which the two (clonal) insertions were located. Significantly, these two fusions yielded the highest AP activities. It seems likely that this proposed transmembrane domain results in the C-terminal position of PsiB being exposed in the periplasm, but there were no special features in the vicinity of the other two fusions (Fig. 3).

One striking feature that emerged from the studies reported here related to the response of strains with multicopy $p s i A$ to the optical brightener calcolluor. As stated, colonies of $R$. leguminosarum do not stain normally with this dye. This is surprising, since calcofluor might be predicted to bind to the glucan backbone of the bacterial LPS. In contrast, the succinoglycan EPS of $\mathrm{R}$. meliloti stains brightly with calcofluor when viewed under UV light. Strains of $\mathrm{R}$. leguminosarum containing multi-copy psi $A$ are non-mucoid (Borthakur et al., 1985) and the supernatants of cultures of such strains contain no detectable EPS (M. A. Jones, unpublished; V. Morris, personal communication). It would seem paradoxical, therefore, that strains with multi-copy psi actually stain with calcofluor when the wild-type does not.

We do not know the reason for this phenomenon, but we have speculated that the following mechanism might be responsible. We had suggested that over-production of PsiA caused accumulation of the protein in the inner bacterial membrane and that this somehow jammed the biosynthesis and/or export of EPS (Borthakur et al., 1986). This inhibition can be overcome by presence of multi-copy pss $A$, whose product is also associated with the inner membrane and which may physically interact with PsiA. We tentatively suggest that, although nonmucoid, strains with cloned psi $A$ have the capacity to make EPS, but that the (perhaps) nascent molecules may be 'trapped' in the inner membrane and so are not exported. We further suggest that the secondary (or tertiary) structure of these forms of EPS differs from that of the wild-type $R$. leguminosarum such that they can bind calcofluor, unlike those of the wild-type. It is certainly evident that whatever the identity of the staining material, it requires a functional $p s s, A$ gene, since mutations in $p s s A$ abolish this phenomenon. These speculations imply that the ability of calcofluor to bind to polymeric glucans and to fluoresce with UV light may depend not only on primary chemical structure, but also to higher orders of molecular structure.

\section{ACKNOWLEDGEMENTS}

This work was funded by the Agricultural and Food Research Council. We are very grateful to A. P. Cavill for skilled technical assistance.

\section{REFERENCES}

Bagdasarian, M., Lurz, R., Ruckert, B., Franklin, F. C. H., Bagdasrian, M. M., Frey, J. \& Timmis, K. T. (1981). Specific-purpose plasmid cloning vectors. II. Broad host range, high copy RSF1010derived vectors and a host-vector system for gene cloning in Pseudomonas. Gene 16, 237-247.

Beringer, J. E. (1974). R factor transfer in Rbizobium leguminosarum. $J$ Gen Microbiol 83, 188-198.

Borthakur, D. (1986). Molecular genetics of exopolysaccharide synthesis in Rhizobium. PhD thesis, University of East Anglia, UK.

Borthakur, D. \& Johnston, A. W. B. (1987). Sequence of psi a gene on the symbiotic plasmid of Rhizobium pbaseoli which inhibits exopolysaccharide synthesis and nodulation and demonstration that its transcription is inhibited by $p s r$, another gene on the symbiotic plasmid. Mol \& Gen Genet 207, 149-154.

Borthakur, D., Downie, J. A., Johnston, A. W. B. \& Lamb, J. W. (1985). psi, a plasmid-linked Rbizobium phaseoli gene that inhibits exopolysaccharide production and which is required for symbiotic nitrogen fixation. Mol \& Gen Genet 200, 278-282.

Borthakur, D., Barber, C. E., Lamb, J. W., Daniels, M. J., Downie, J. A. \& Johnston, A. W. B. (1986). A mutation that blocks exopolysaccharide synthesis prevents nodulation of peas by $R b i$ zobium leguminosarum but not of beans by $R$. phaseoli and is corrected by cloned DNA from Rhizobium or the phytopathogen Xanthomonas. Mol \& Gen Genet 203, 320-323.

Borthakur, D., Barker, R. F., Latchford, J.W., Rossen, L. \& Johnston, A. W. B. (1988). Analysis of pss genes of Rhizobium leguminosarum required for exopolysaccharide synthesis and nodulation of peas: their primary structure and their interaction with $p s i$ and other nodulation genes. Mol \& Gen Genet 213, 155-162.

Buchanan-Wollaston, A. V., Beringer, J. E., Brewin, N. J., Hirsch, P. R. \& Johnston, A. W. B. (1980). Isolation of symbiotically defective mutants in Rbizobium leguminosarum by insertion of the transposon Tn5 into a transmissible plasmid. Mol \& Gen Genet 178, 185-190.

Davis, E. O. \& Johnston, A. W. B. (1990a). Analysis of three nodD genes in Rbizobium leguminosarum biovar phaseoli; nodD1 is preceded by nolE, a gene whose product is secreted from the cytoplasm. Mol Microbiol 4, 921-932.

Davis, E. O. \& Johnston, A. W. B. (1990b). Regulatory functions of the three nodD genes of Rhizobium leguminosarum biovar phaseoli. Mol Microbiol 4, 933-941.

Downie, J. A. \& Johnston, A. W. B. (1988). Nodulation of legumes by Rbizobium. Plant Cell Environ 11, 403-412.

Finan, T. M., Kunkel, B., de Vos, G. F. \& Signer, E. R. (1986). Second symbiotic megaplasmid in Rbizobium meliloti carrying 
exopolysaccharide and thiamine synthesis genes. $J$ Bacteriol 167, 66-72.

Fisher, R. F. \& Long, S. R. (1992). Rhizobium - plant signal exchange. Nature 357, 655-660.

Gray, J. X. \& Rolfe, B. G. (1990). Exopolysaccharide production in Rhizobium and its role in invasion. Mol Microbiol 4, 1425--1431.

Gray, J. X., Djordjevic, M. A. \& Rolfe, B. G. (1990). Two genes that regulate exopolysaccharide production in Rbizobium sp. strain NGR234: DNA sequences and resultant phenotypes. J Bacteriol 172, 193 203

Hawkins, F. K. L. \& Johnston, A. W. B. (1988). Transcription of a Rbizobium leguminosarum biovar phaseoli gene needed for melanin synthesis is activated by nif $A$ of Rbizobium and Klebsiella pneumoniae. Mol Microbiol 2, 331-337.

Hotter, G. S. \& Scott, D. B. (1991). Exopolysaccharide mutants of Rhizobium loti are fully effective on a determinate nodulating host but are ineffective on an indeterminate nodulating host. $J$ Bacteriol 173, 851-859.

Hynes, M. F., Simon, R., Muller, P., Niehaus, K., Labes, M. \& Puhler, A. (1986). The two megaplasmids of Rhizobium meliloti are involved in the effective nodulation of alfalfa. Mol \& Gen Genet 202, 356-362.

Lamb, J. W., Hombrecher, G. \& Johnston, A. W. B. (1982). Plasmiddetermined nodulation and nitrogen-fixation abilities in Rhizobium phaseoli. Mol \& Gen Genet 186, 449-452.

Lamb, J. W., Downie, J. A. \& Johnston, A. W. B. (1985). Cloning of the nodulation (nod) genes of Rbizobium phaseoli and their homology to R. leguminosarum nod DNA. Gene 34, 235-241.

Latchford, J. W., Borthakur, D. \& Johnston, A. W. B. (1991). The products of Rhizobium genes, psi and pss, which affect exopolysaccharide production, are associated with the bacterial cell surface. Mol Microbiol 5, 2107-2114.
Leigh, J. A. \& Coplin, D. L. (1992). Exopolysaccharides in plantbacterial interactions. Annu Rev Microbiol 46, 307-346.

Leigh, J. A., Signer, E. R. \& Walker, G. C. (1985). Exopolysaccharide-deficient mutants of Rbizobium meliloti that form ineffective nodules. Proc Natl Acad Sci, US A 82, 6231-6235.

Leigh, J. A., Reed, J. W., Hanks, J. F., Hirsch, A. M. \& Walker, G. C. (1987). Rhizobium meliloti mutants that fail to succinylate their calcofluor-binding exopolysaccharide are defective in nodule invasion. Cell 51, 579-587.

Long, S., Reed, J. W., Himawan, J. \& Walker, G. C. (1988). Genetic analysis of a cluster of genes required for synthesis of the calcofluorbinding exopolysaccharide of Rbizobium meliloti. J Bacteriol 170, 4239-4248.

Mimmack, M. L., Hong, G. F. \& Johnston, A. W. B. (1994). Identification of $p s r A$, a gene on the sym plasmid of Rbizobium leguminosarum biovar phaseoli which inhibits transcription of the $p s i$ genes. Microbiology 140, 455-461.

Reed, J. W., Capage, M. \& Walker, G. C. (1991). Rhizobium meliloti exoG and exol mutations affect the ExoX-ExoY system for modulation of exopolysaccharide production. $J$ Bacteriol 173, 3776-3788.

Sambrook, J., Fritsch, E. F. \& Maniatis, T. (1989). Molecular Cloning. A Laboratory Manual. Cold Spring Harbor, NY: Cold Spring Harbor Laboratory.

Tulley, R. E. \& Terry, M. E. (1985). Decreased exopolysaccharide synthesis by anaerobic and symbiotic cells of Bradyrbizobium japonicum. Plant Pbysiol 79, 445-450.

Wood, W. B. (1966). Host specificity of DNA produced by E. coli bacterial mutations affecting the restriction and modification of DNA. J Mol Biol 16, 118-133.

Received 23 August 1993; revised 23 November 1993; accepted 1 December 1993. 